\title{
Novelties and New Potentials in the Clinical Application of SPECT/CT Imaging
}

Sarolta Szekeres*, Erzsébet Schmidt, Zsuzsanna Szabó, Zsuzsanna Bán and Katalin Zámbó

Department of Nuclear Medicine, University of Pécs Clinical Center, Hungary

*Corresponding author: Sarolta Szekeres, Department of Nuclear Medicine, University of Pécs Clinical Center, Hungary, Tel: 003672535841; E-mail: sacc71@gmail.com

Received date: Jun 29, 2016; Accepted date: August 05, 2016; Published date: August 09, 2016

Copyright: ( 2016 Szekeres S, et al. This is an open-access article distributed under the terms of the Creative Commons Attribution License, which permits unrestricted use, distribution, and reproduction in any medium, provided the original author and source are credited.

\begin{abstract}
The application of hybrid devices that is fused technologies is getting more and more important in the field of imaging diagnostics. The greatest advantage of this method is the combined use of several modalities, which can provide data about the morphological, functional and possibly molecular changes in different diseases simultaneously. In this paper the potentials, advantages and applicabilities of SPECT/CT (single photon emission computer tomograph/computer tomograph) are summarized mainly in oncological diseases but in other diseases as well. Multimodality devices detecting functional and morphological abnormalities simultaneously increase the specificity and diagnostic accuracy of nuclear medicine methods and therefore the effectiveness of therapy too.
\end{abstract}

Keywords: SPECT/CT; Fusion imaging; Molecular diagnostics; Specific radiotherapy

\section{Introduction}

In the past decade different areas of diagnostic imaging showed an abrupt development. At the same time, hybrid imaging i.e. combined use of different modalities when performing fusion image has become increasingly important $[1,2]$. Interestingly, in our country PET/CT (positron emission tomograph/computer tomograph) devices began to work earlier than SPECT/CT- that can be used in "traditional" isotopediagnostic methods the latter looking back on just a 7 year long period. The first SPECT/CT hybrid device of Hungary was installed at the Department of Nuclear Medicine, University of Pécs.

SPECT/CT is a combined device consisting of two imaging modalities that function simultaneously producing fusion image which allows for a more accurate simultaneous morphological and functional assessment of lesions during the same examination. The use of SPECT/CT device is particularly advantageous in nuclear oncology where SPECT scan is aimed at detecting mostly soft tissue lesions and the localization and identification of organ involvement is extremely difficult without morphological investigation. However, it can be made much more precise with co-evaluation of SPECT and CT image sections $[3,4]$. In nuclear medicine numerous methods are known that are suitable to detect primary tumours and metastases in different types of cancer. These methods use radiopharmaceuticals accumulating nonspecifically or partly specifically, binding only to certain types of tumour cells. Among the latter several receptor binding molecules are known (meta-iodo-benzyl-guanidine [MIBG], pentetreotide, octreotide, depreotide) which are suitable for the detection of various hormone-producing neuroendocrine tumours when marked with gamma radiation isotopes. Their sensitivity and specificity is also high.

In addition, it is possible to use hybrid imaging techniques in diagnosing bone lesions of unknown or uncertain origin. Bone scintigraphy with adjunct SPECT/CT significantly improves the specificity, the exact classification of lesion etiology $[5,6]$.
Regional lymph node status is an important prognostic factor in gynecologycal neoplasms. Scintigraphy of sentinel lymph nodes has become an important part of surgical intervention in vulva tumours $[7,8]$, however, there is a demand for using the method in the treatment of early-stage cervical and endometrial cancers as well. The conventional planar scan supplemented by a SPECT/CT image provides a more precise orientation in the pelvic lymphatic regions [9].

In the last few decades the incidence of thyroid cancers has doubled worldwide. This is partly due to improvement and accessibility of testing methods thus more and more cases earlier than ever are detected, however, the incidence of the disease is actually increasing. Up to $10 \%$ of cold nodules not accumulating radiopharmaceuticals detected by isotope or ultrasound examinations in the thyroid gland can be thyroid cancer. The therapy is based on near-total or total thyroidectomy followed by radioiodine remnant ablation in differenciated cancers or radioiodine treatment of metastases [10]. Using SPECT/CT hybrid device with an examination of relatively small exposure performed after high dose radiotherapy of thyroid cancers immediate additional information is obtained that can be crucial concerning the etiology of lesions, stage of the disease and also the further treatment.

SPECT/CT device can also be used when performing cardiological examinations to carry out absorption correction through which a reduction in false positive cases and an increase in diagnostic accuracy can be achieved [11]. When using an at least 16 slice multislice CT scan narrowing of the coronary arteries and the consequent microcirculatory alterations in the heart muscle can be studied simultaneously.

Therefore SPECT/CT is a modern diagnostic imaging device which improves the accuracy of diagnostic tests, helps the precise localization of lesions and has an important role in planning specific radiotherapy and also in monitoring its effects. It reduces the number of patient visits to health care institutions which is not an insignifacant factor in economic terms either. 


\section{Significance of Molecular Imaging}

MIT's Technology Review in 2003 described molecular imaging as "one of the ten developing technologies that will change the world" [12]. The aim of the young, fast developing biomedical research discipline is to serve the development of biology and medicine through in vivo observation of molecular events in normal and pathological processes taking place in cells. It combines the modern devices of molecular and cell biology with the most advanced technical technology. The discipline was created by the fusion of borderline areas of molecular biology, chemistry, medicine, pharmacology, medical physics, biomathematics, bioinformatics and numerous fields of imaging technology. Molecular imaging deals with visualization, characterization and quantification of biological processes at cellular and sub-cellular level taking place in the living organism [13].

In nuclear medicine there are numerous examination options to detect various diseases. Methods are based on altered biochemical processes of damaged organs. Scintigraphic investigations of individual organs indirectly indicate the functional change, tissue damage that is created by the development of the disease. In many cases due to the location and small size of the tumour other imaging techniques are not suitable to detect the lesion. Methods of nuclear medicine provide functional data regarding the biochemical status of individual cancers that are highly significant from the point of view of early detection, staging, restaging, therapy planning, control and first of all patient's life expectancy. However the more specific the radiopharmaceutical binding the fewer other structures emerge and the more difficult is the precise localization. Multimodality devices are trying to solve this problem of which SPECT/CT is now well-known in oncological diagnostics.

\section{Benefits of Multimodality Devices}

With the development of imaging diagnostics the fusion, the combined feasibility of mapping, functioning on different principles has come to the fore. Hungary is now operating five PET/CT devices while international literature reported about PET/MR fusion devices too [14].

Information obtained during functional imaging typically provides limited anatomic-morfological information. In the case of multimodality devices the radiographic CT device is located on the same gantry as the section suitable for functional imaging thus allowing for automatic image fusion. The combined visualization of functional and anatomic information provides significant benefits in human diagnostics [13].

SPECT/CT is a combined device creating two imaging modalities simultaneously. The essence of the resulting fusion imaging is that the CT image detecting structural changes in the body is performed at one time without removing the patient. In this CT image functional changes based on testing principles of nuclear medicine can be welllocalized that is abnormal or normal conditions of metabolism, microcirculation, hormone synthesis, receptor distribution, etc. Thanks to the new tomographic imaging device doctors are able to examine molecular processes taking place in the patient with millimeter resolution accuracy, the same applies to the detection of the exact anatomic location [1,2]. SPECT is one of the most sensitive devices among molecular in vivo imaging technologies. With the help of gamma photons emitted from the patient sectional images of living systems can be produced without disrupting their unity. SPECT device is suitable for examining a number of different disease processes, using different radiopharmaceuticals for example increased metabolism of metastatic bone lesions, blood supply to the brain, lungs or coronary arteries, destruction of certain cells of parenchymatous organs etc. can be studied. It allows for faster and more precise diagnostics, for monitoring the progression of the disease for a longer time and also for the evaluation of the efficacy of therapeutic interventions.

Using SPECT/CT device investigations well-known in routine diagnostics may be performed, however with an additional CT scan investigation accuracy increases significantly and it is possible to localize the lesion immediately [15]. Another significance of SPECT/CT device is the possibility of applying absorption and motion-correction. Because gamma-rays from patients passing through the tissues are partially absorbed depending on the thickness images resulting in false positivity may be obtained. The application of absorption correction of X-ray through elimination of artifacts, the three-dimensional (3D) and four-dimensional (4D) image display or the possibility of 3D printing in the near future significantly increases and will increase the specificity of diagnostics and also the effectiveness of the therapy $[1,2,16]$.

\section{Clinical Applications of SPECT/CT}

The main indications for use of the device:

1. Direct scintigraphies: Using radiopharmaceuticals accumulating directly in the tumours,

a. Specific: somatostatin receptor (111 In-pentetreotide [Octreoscan] 99m Tc-depreotide [NeoSpect]), adrenergic receptor (131-I, 123-I-meta-iodo-benzyl-guanidine [MIBG]) bind, participant (131-I Na-iodine therapy, 131-I-methyl-nor-cholesterol) radiopharmaceuticals in hormone synthesis.

b. Nonspecific: 99m-Tc-diphosphonate methyl [MDP], Tl-201 chloride, Tc-99m methoxy isobutyl isonitrile [MIBI] 99m Tctetrofosmin, $67 \mathrm{Ga}$-citrate

2. Completion as indirect scintigraphies (bone, brain perfusion, cerebral receptor, liver, thyroid, parathyroid, sentinel lymph node, etc.) in addition to,

3. Follow-up therapy, the specific efficacy of radiotherapy forecast

4. Application of attenuation correction.

\section{Direct imaging}

The main advantages of the application of SPECT/CT device were found during specific oncological examinations. The aim of examinatons to detect some typical functional characteristics of tumour cells is to achieve that possibly only cancer cells and tissues are visualized during imaging in nuclear medicine. Although generally this is not fully achieved because some organs are usually visualized physiologically too nevertheless it is difficult to orientate onself in the body during these investigations and is not easy to localize the lesion accurately. On the other hand, regarding the patient's prognosis it is not all the same whether a malignancy is situated within the boundaries of an organ or it has already spread to its environment. In such cases it is a great help when the isotope image can be placed on a CT image showing the anatomic structure accurately thus creating fusion visualization $[3,4]$.

The investigation of pheocromocytoma and neuroblastoma countaining adrenergic receptors, lung tumors and gastrointestinal 
Page 3 of 6

carcinoids expressing somatostatin receptors as well as the detection of increased hormone production in Cushing's syndrome are used routinelly both at our department and according to the international literature.

\section{Adrenergic receptor investigation}

Gross at al. [17] reported on isotope investigation options of endocrine organ lesions in 1984. A number of catecholamine producing neuroendocrine tumour cells accumulate iodine-123 or iodine-131 labelled MIBG administered intravenously. Pheochromocytoma and neuroblastoma are the most common indications for the test. Hugosson at al. [18] compared several isotopic and radiological imaging techniques in children with neuroblastoma and came to the conclusion that CT and MR scans are the most useful devices in the accurate detection of the relationship between local lesions and certain organs whereas isotope methods are more suitable in diagnosing metastatic diseases.

They also thought that the application of SPECT/CT device in these investigations significantly increased diagnostic accuracy. Based on data provided by Rozovsky et al. [19] in $89 \%$ of cases whose evaluation is uncertain when using other methods fusion technique improves diagnostic accuracy?

Our department has been performing these investigations routinely for more than 10 years. Previously assessment was performed based on planar and SPECT images while during the past seven years in the case of every patient SPECT/CT images have been made of multiple regions (Figure 1). Data processing involved the analysis of fourtythree MIBG investigations. Two independent specialists carried out the assessment based on only planar and SPECT images and then considering SPECT/CT recordings as well.

Our results show that SPECT/CT recordings made final evaluation more precise in $18.6 \%$ of cases. Consequently fusion imaging techniques are extremely sensitive indicators of the presence of tumour tissue and at the same time precisely localize the tumour. This is particularly useful in cases of multifocal lesions and when an immediate obvious accumulation of radiopharmaceuticals, attracts attention to small alteratious in CT images.
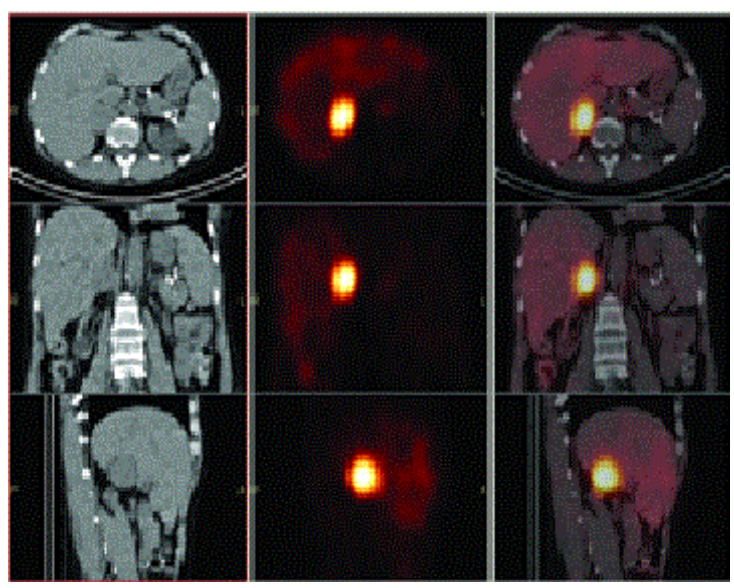

Figure 1: 123-I-MIBG SPECT/CT examination- typical phaeocromocytoma on the right side.

\section{Somatostatin receptor investigation}

Hormone-producing tumours of the gastrointestinal tract and certain lung cancer cells contain large quantities of somatostatine receptors thus these tumours can be detected using radiopharmateuticals binding to them. Five diffent receptor subtypes are known of which subtype two binds to 111-indium-penetetreotide molecules subtypes 2, 3 and 5 bind to $99 \mathrm{mTc}$-depreotide molecule. Benefits of somastostatine receptor scintigraphy were reported in the early 90s [20] in the detection of different hormone-producing tumours such as carcinoid tumours, certain pancreatic and pituitary tumours and also various brain tumours. Based on the histologycally proven results of Cwika et al. [21] the diagnostic accuracy of 111indium-octreotide scan in neuroendocrine tumours was significantly better than that of CT investigations partcularly in the case of multiple lesions.

Therefore the use of the former is recommended when neuroendocrine tumours are suspected. Fusion imaging produced by SPECT/CT device significantly improves diagnostic accuracy of the investigation in these tumours too. The results of Perri et al. [22] show that octreotide SPECT/CT accuracy reaches $94.7 \%$ in detecting and localizing lesions, which is significantly higher than SPECT alone, with its only $45.6 \%$ of accuracy.

At our department the results of somatostatine receptor investigations supplemented by fourtyeight SPEC/CT images were analyzed. Data processing was similar to that of in MIGB investigations. Based on these results fusion imaging clarified the diagnosis confirmed histologically later and also tumour location in $29.1 \%$ of cases.

Scintigraphic method with an adjunct fusion technique is well applicable in these cases too in the detection and localization of tumours, in indentifying their connection with other organs, in signalling distant metastases, in monitoring the effectiveness of therapy and also in detecting recurrences (Figure 2).

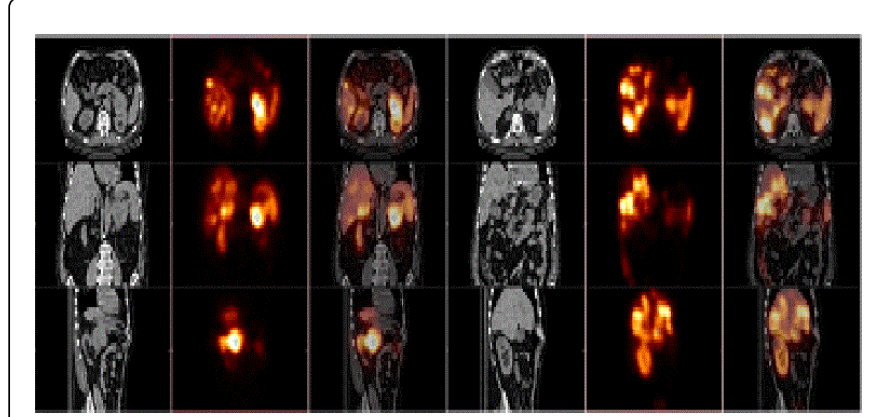

Figure 2: 111-In-Octreotide SPECT/CT examination- carcinoid tumour in the pancreas and its metastases in the liver.

\section{Investigations based on non-specific accumulation}

Concerning lesions accumulating certain radiopharmaceuticals non-specifically a huge number of parathyroid investigations are performed at our department using dual tracer technique. Thyroid tissue can be visualized with the help of $99 \mathrm{~m}$-technetium-pertecnetate. $99 \mathrm{~m}-\mathrm{Tc}-\mathrm{MIBI}$, on the other hand, accumulates in proliferative tissues rich in mitochondria thus making detectable thyroid and parathyroid adenomas. Withdrawing the two digital images only the parathyroid 
adenoma becomes visible. Fusion SPECT/CT image is a great help in its localization, surgical intervention and perfect removal (Figure 3) [23].

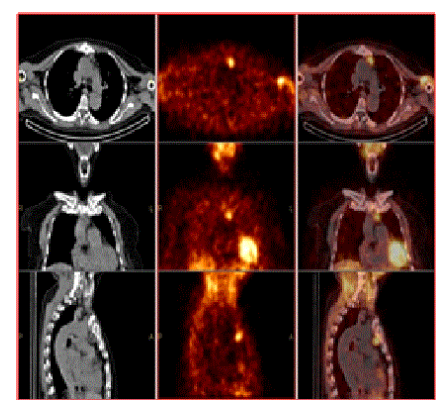

Figure 3: 99m-Tc-MIBI SPECT/CT examination- two ectopic parathyreoid adenomas behind the sternum.

\section{Indirect scintigraphies}

Additional SPECT/CT images can be performed during the examination of every organ provided we have some prior information about certain morphological changes that can affect the assessment of isotope investigations or there is a need to confirm such alterations. In cases of cerebral perfusion and cerebral presynaptic dopamine receptor investigations CT scans are performed routinely too to show anatomic structures or maybe to facilitate MRI coregistration. The situation is similar in liver examinations (colloidal scintigraphy, blood-pool testing) when the size, position, any visible CT morphological alterations of abdominal organs can be of great help during the wording of the last finding. Performing additional SPECT/CT images in the case of bone scintigraphy investigations can be extremly useful in those not infrequent cases when we have to differenciate between degenerative and metastatic bone processes equally causing an increase in bone metabolism as confirmation or exclusion of distant metastases is crucial concerning primary tumour therapy (Figure 4). Equally useful information is obtained during SPECT/CT scintigraphy of any organ when through exact localization structural changes or even the lack of them underlying sensitive but non-specific isotope changes can be detected [24].

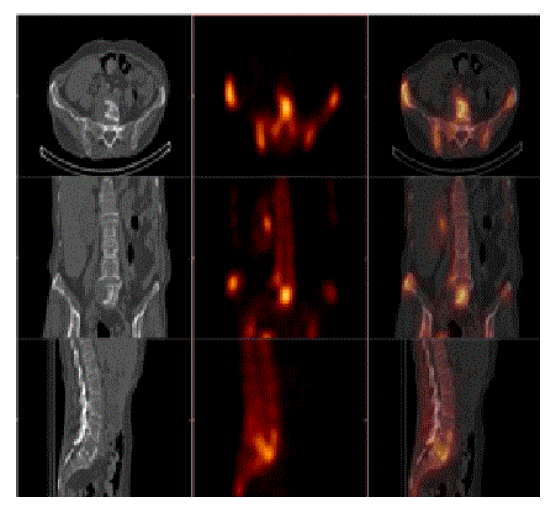

Figure 4: SPECT/CT examination with 99m-Tc-MDP- lytic metastasis of prostatic cancer in the lumbal spine (L.V. vertebra).
Cooperation with Positron Diagnostics Ltd. of Budapest we had the opportunity to compare the bone scan results of 27 patients who, as part of their onclogical check-up underwent both bone scintigraphy with an additional SPECT/CT scan and PET/CT imaging. In $48 \%$ of patients both SPECT/CT and PET/CT methods gave negative results concerning bone metastases, in $22 \%$ both methods detected metastases whereas the number of uncertain cases amounted to $30 \%$. The difference may be due to the fact that the two methods of nuclear medicine detect pathological processes using different basic mechanisms however, the detection of bone metastases using bone scintigraphy with additional SPECT/CT investigation is a considerably cheaper procedura than PET/CT.

In gynecological tumours planar and SPECT/CT examinations are performed to localize sentinel lymph nodes, these nodes are removed using intraoperative gamma probes. In each patient this is followed by pelvic lymphadenectomy and histological examination of the lymph nodes (Figure 5). Our method seems to be a succesful and useful procedure in the treatment of early-stage cervical and endometrial cancer patients thereby making it possible to avoid any unnecessary extended surgeries in the future [25]. SPECT/CT imaging is also suitable for examining sentinel lymph nodes of other tumours (breast cancer, malignant melanoma, penile, prostate carcinoma) if there are problems with localization.

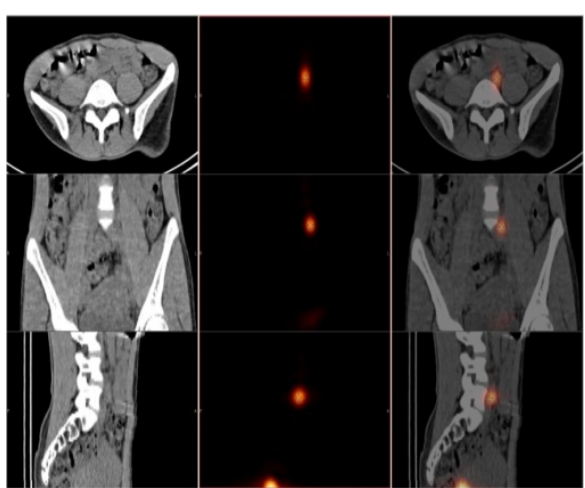

Figure 5: 99m-Tc-Sentiscinte SPECT/CT examination- The patient has a second-stage of cervical adenocarcinoma. The sentinel lymph node is located in the left parailiacal region (in taunt position).

\section{Planning Specific Radiotherapy, Therapy Monitoring}

Benefits of fusion receptor investigations are significant not only in diagnostics but they also play a very important role in selecting the applicable radiotherapy and in predicting therapeutic efficacy. Similar to basic principle of diagnostics receptor binding molecules are capable of carrying beta radiating that is suitable for therapy isotopes to tumour cells. Beta radiation is a fast electron leaving the nucleus with a small penetration depth of 1-2 mm- thus it transmits its energy in the tissues and is absorbed. Therefor this therapeutic method destroys only tumour tissues and does not affect intact organs. The therapeutic use of high dose 131-iodine-MIGB and 90-yttrium-octreotide has already been introduced in Europe-the former is available in Hungary, too. However, it is a prerequisite to treatment that cancer cells contain appropriate receptors to facilitate binding. Consequently, the abovementioned receptor scintigraphies indicate the effectiveness of receptor binding as well. If cells contain many receptors the intensity of 
accumulation high resulting in an equally intense binding of therapeutic radiopharmaceuticals i.e. therapeutic effect. Neuroendocrine tumours accumulate MIGB and octreotide but not so same degree. Esfandiari et al. [26] proposed the selection of the therapy on the basis of the binding of the two diagnostic pharmaceuticals in patients with malignant pheochromocytoma. O' Dorisio et al. [27] emphasized the high sensitivity of combined functional and anatomic imaging in diagnostics, its significant perdictive value in the prognosis of therapeutic effect and also its high sensitivity in monitoring therapeutic effect.

At our department large numbers of radiotherapy using 131-iodinesodium-iodide are carried out in patients with thyroid cancer in whom the detection of partly the remnant in the neck partly distant metastases is essential both in judging effectiveness of the therapy and determining further treatment options. Total body image performed after high-dose radioiodine therapy of thyroid cancers is a useful method to detect the remaining thyroid tissue as well as cervical and distant iodine accumulated metastases after near-total or total thyreoidectomy (Figure 6). However, on total body images anatomic boundaries are not visible, structures accumulating iodine are difficult to localize. Images are often disturbed by the appearance of ectopic thyroid tissue, of organs excreting iodine physiogically (mucous membranes of the nose and oral cavitiy, salivary glands, liver, stomach, intestines, urinary bladder) and also of potential contamination of the skin [28]. SPECT/CT hybrid device is suitable for further examination of unidentified radioiodine accumulations. It performs isotope tomography and low-dose native CT image simultaneously without removing the patient, which when fused makes the exact localization of accumulations possible [29].

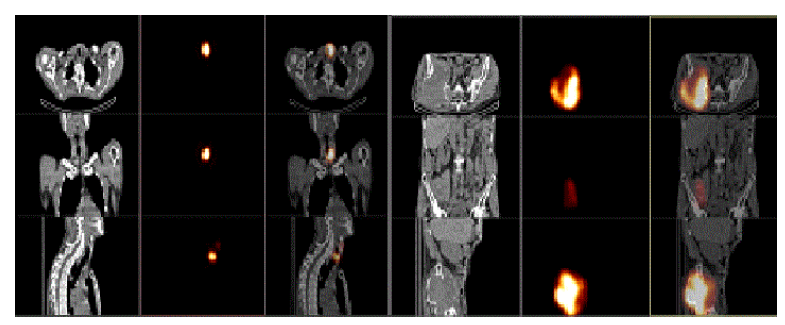

Figure 6: SPECT/CT examination after high dose 131-I-NaI therapy in patient with follicular thyreoid cancer. Recurrent tumour on the left side of the thyreoid gland with extended bone and soft tissue metastasis.

\section{Application of Attenuation Correction}

In cardiology the significance of SPECT/CT method is provided primarily by the possibility of applying absorption correction. In these investigations the number of false positive results is relatively high because bulky breasts in females make the assessment of anterior wall of the left ventricle uncertain whereas in males higher location of the diaphragm produces difficulties in evaluating the inferior wall of the left ventricle. The absorption of transmission $\mathrm{x}$-rays in tissues provides an opportunity to judge their thickness and also to correct gamma radiation tissue absorption. This can reduce the number of false positive inevstigation results thus increasing investigation specificity.
Using MSCT (at least 16-slice CT) we can obtain simultaneous data about myocardial perfusion, degree of coronary stenosis and calcium score as well [30].

\section{Conclusion}

SPECT/CT is a useful device in everyday routine diagnostics of nuclear medicine which through creating functional and morphological image fusion-improves the potential of precise localization of lesions, brings doctors closer to etiology of diseases, helps to eliminate inaccurities of absorbtion and motion in images received thus, with all these, improving the specificity and sensitivity of investigations and the effectiveness of the applied therapy.

\section{References}

1. Schäfers KP, Stegger L (2008) Combined imaging of molecular function and morphology with PET/CT and SPECT/CT: image fusion and motion correction. Basic Res Cardiol 103: 191-199.

2. Ferro-Flores G, de Murphy CA (2007) Current developments in SPECT/CT systems using 99mTc-radiopharmaceuticals. Rev Invest Clin 59: 373-381.

3. Schillaci O, Simonetti G (2004) Fusion imaging in nuclear medicine-applications of dual-modality systems in oncology. Cancer Biother Radiopharm 19: 1-10.

4. Chowdhury FU, Scarsbrook AF (2008) The role of hybrid SPECT-CT in oncology: current and emerging clinical applications. Clin Radiol 63: 241-251.

5. Horger M, Bares R (2006) The role of single-photon emission computed tomography/computed tomography in benign and malignant bone disease. Semin Nucl Med 36: 286-294.

6. Römer W, Nömayr A, Uder M, Bautz W, Kuwert T (2006) SPECT-guided CT for evaluating foci of increased bone metabolism classified as indeterminate on SPECT in cancer patients. J Nucl Med 47: 1102-1106.

7. Zámbó K, Schmidt E, Hartmann T, Kornya L, Dehghani B, et al. (2002) Preliminary experiences with sentinel lymph node detection in cases of vulvar malignancy. Eur J Nucl Med Mol Imaging 29: 1198-1200.

8. Zámbó K, Koppán M, Paál A, Schmidt E, Tinneberg HR, et al. (2003) Sentinel lymph nodes in gynaecological malignancies: frontline between TNM and clinical staging systems? Eur J Nucl Med Mol Imaging 30: 1684-1688.

9. Díaz-Feijoo B, Pérez-Benavente MA, Cabrera-Diaz S, et al. (2011) Change in clinical management of sentinel lymph node location in early stage cervical cancer: the role of SPECT/CT. Gynecol Oncol 120: 353-357.

10. Bombardieri E, Pacini F (2009) Radioiodine therapy a successful tool in treating thyroid cancer. Foreword. Q J Nucl Med Mol Imaging 53: 439.

11. Akincioglu C, Belhocine T, Gambhir S, Romsa J, Chouraiki G, et al. (2008) Complementary roles of low-dose SPECT-CT and high-resolution volume CT for detection of coronary artery disease. Clin Nucl Med 33: 285-287.

12. http://www.technologyreview.com/Infotech/13060/page7/

13. Illés M (2007) Submillimeter-resolution molecular imaging. IME 6: 51-55.

14. Steinmetz H, Huang Y, Seitz RJ, Knorr U, Schlaug G, et al. (1992) Individual integration of positron emission tomography and highresolution magnetic resonance imaging. Cereb Blood Flow Metab 12: 919-926.

15. Patel CN, Chowdhury FU, Scarsbrook AF (2008) Clinical utility of hybrid SPECT-CT in endocrine neoplasia. AJR Am J Roentgenol 190: 815-824.

16. Li G, Citrin D, Camphausen K, Mueller B, Burman C, et al. (2008) Advances in $4 \mathrm{D}$ medical imaging and $4 \mathrm{D}$ radiation therapy. Technol Cancer Res Treat 7: 67-81.

17. Gross MD, Shapiro B, Thrall JH, Freitas JE, Beierwaltes WH (1984) The scintigraphic imaging of endocrine organs. Endocr Rev 5: 221-281. 
Citation: Szekeres S, Schmidt E, Szabó Z, Bán Z, Zámbó K (2016) Novelties and New Potentials in the Clinical Application of SPECT/CT Imaging. J Nucl Med Radiat Ther 7: 298. doi:10.4172/2155-9619.1000298

Page 6 of 6

18. Hugosson C, Nyman R, Jorulf H, McDonald P, Rifai A, et al. (1999) Imaging of abdominal neuroblastoma in children. Acta Radiol 40: 534-542.

19. Rozovsky K, Koplewitz BZ, Krausz Y, Revel-Vilk S, Weintraub M, et al. (2008) Added value of SPECT/CT for correlation of MIBG scintigraphy and diagnostic CT in neuroblastoma and pheochromocytoma. AJR Am J Roentgenol 190: 1085-1090.

20. Lamberts SW, Bakker WH, Reubi JC, Krenning EP (1990) Somatostatin receptor imaging in vivo localization of tumors with a radiolabeled somatostatin analog. J Steroid Biochem Mol Biol 37: 1079-1082.

21. Cwika JB, Buscombe JR, Mielcarek WA, Caplin ME, Watkinson AJ, et al. (2001) Comparison of functional imaging and standard CT in evaluation of disease extent in patients with tumours showing neuroendocrine features. Nucl Med Rev Cent East Eur 4: 27-33.

22. Perri M, Erba P, Volterrani D, Lazzeri E, Boni G, et al. (2008) OctreoSPECT/CT imaging for accurate detection and localization of suspected neuroendocrine tumors. Q J Nucl Med Mol Imaging 52: 323-333.

23. Rubello D, Casara D, Fiore D, Muzzio P, Zonzin G, et al. (2002) An ectopic mediastinal parathyroid adenoma accurately located by a singleday imaging protocol of Tc-99m pertechnetate-MIBI subtraction scintigraphy and MIBI-SPECT-computed tomographic image fusion. Clin Nucl Med 27: 186-190.

24. Helyar V, Mohan HK, Barwick T, Livieratos L, Gnanasegaran G, et al. (2010) The added value of multislice SPECT/CT in patients with equivocal bony metastasis from carcinoma of the prostate. Eur J Nucl Med Mol Imaging 37: 706-713.
25. Buda A, Elisei F, Arosio M, Dolci C, Signorelli M, et al. (2012) Integration of hybrid single-photon emission computed tomography/computed tomography in the preoperative assessment of sentinel node in patients with cervical and endometrial cancer: our experience and literature review. Int J Gynecol Cancer 22: 830-835.

26. Esfandiari NH, Shulkin BL, Bui C, Jaffe CA (2006) Multimodality imaging of malignant pheochromocytoma. Clin Nucl Med 31: 822-825.

27. O'Dorisio MS, Khanne G, Bushnell D (2008) Combining anatomic and molecularly targeted imaging int he diagnosis and surveillance of embryonal tumors of the nervous and endocrine systems in children. Cancer Metastasis Rev 27: 665-677.

28. Shapiro B, Rufini V, Jarwan A (2000) Artifacts, anatomical and physiological variants, and unrelated diseases that might cause falsepositive whole-body 131I scans in patients with thyroid cancer. Semin Nucl Med 30:115-132.

29. Kohlfuerst S, Igerc I, Lobnig M, Gallowitsch HJ, Gomez-Segovia I, et al (2009) Posttherapeutic 131I SPECT-CT offers high diagnostic accuracy when the findings on conventional planar imaging are inconclusive and allows a tailored patient treatment regimen. Eur J Nucl Med Mol Imaging 36: 886-893.

30. Almoudi M, Sun ZH (2012) A head-to-head comparison of the coronary calcium score by computed tomography with myocardial perfusion imaging in predicting coronary artery disease. J Geriatr Cardiol 9: 349-354. 